\title{
Signal Properties of Spaceborne Squint-Mode SAR
}

\author{
Gordon W. Davidson, Member, IEEE, and Ian Cumming, Member, IEEE
}

\begin{abstract}
This paper presents signal properties of spaceborne, strip-map SAR for very large squint angles. Doppler centroid, azimuth bandwidth, and exposure time are derived in terms of the platform attitude and elevation angle. This allows a description of the Doppler centroid variation with range and terrain height, and explains a possible range dependence of azimuth bandwidth at high squint. The yaw and pitch required to minimize Doppler centroid and azimuth bandwidth variation are derived, and residual Doppler centroid variation in the presence of antenna pointing errors is presented. The SAR constraint on the relationship between swath width and azimuth bandwidth is revisited for high squint, and is used to present a fundamental limit on squint angle. Finally, processing considerations arising from the shape of the two-dimensional (2-D) spectrum with high squint are discussed.
\end{abstract}

\section{INTRODUCTION}

$\mathbf{S}$ IGNAL properties of spaceborne, strip-map synthetic aperture radar (SAR), such as Doppler centroid and azimuth bandwidth, are well understood for the conventional case in which the antenna is pointed nearly perpendicular to the flight path. In squint mode, the antenna is pointed forward or backward from the perpendicular position by a squint angle that is as much as several tens of degrees. Squint mode has been used in airborne platforms, and for this case Doppler properties are typically described by the orientation of the antenna footprint compared to iso-Doppler lines on a flat surface [1]. However, signal properties are difficult to quantify with this approach in the case of a general earth geometry as with a spaceborne platform and in the case that terrain height variation is to be taken into account.

In this paper we present a general approach to describing high squint SAR signal properties, without small angle approximations, in terms of the platform attitude and elevation angle. This is valid for any orbit and earth geometry, including terrain height, and the effects on signal properties of antenna pointing errors from a desired attitude are readily obtained. In particular, the variation of Doppler centroid with range and terrain height, and a possible range-dependence of azimuth bandwidth, are investigated. Range variation of the Doppler centroid can lead to different wraparound of azimuth frequencies at different ranges, after sampling by the pulse repetition frequency $(\mathrm{PRF})$. This can be accommodated by replication of azimuth spectra [2], but if the variation is large

Manuscript received June 29, 1995; revised August 22, 1996. This work was supported by the B.C. Science Council, the Natural Science and Engineering Research Council of Canada, the B.C. Advanced Systems Institute, and MacDonald Dettwiler and Associates.

G. W. Davidson is with the Advanced Development Group, Glenayre, Vancouver, B.C., Canada (e-mail: gdavidso@glenva.glenayre.com).

I. Cumming is with the Department of Electrical Engineering, University of British Columbia, Vancouver, B.C., Canada V6T 1 Z4.

Publisher Item Identifier S 0196-2892(97)00378-1. it can significantly increase the computation of processing. Doppler centroid variation with terrain height can lead to undersampling and azimuth ambiguities. Finally, the behavior of azimuth bandwidth is important in terms of maintaining a PRF that satisfies the SAR constraint of fitting a received echo between transmitted pulses. It is shown how, with proper attitude steering, Doppler centroid variation is minimized, and typical SAR properties are preserved in squint mode, allowing data to be collected accurately and efficiently processed in the two-dimensional (2-D) frequency domain.

The high squint results are general and therefore provide a thorough understanding of the SAR signal. Furthermore, squint mode has the potential to provide information such as the azimuth angle dependence of backscatter. Since the visibility of structural features on the surface can depend on the viewing angle, this would aid the interpretation of SAR imagery in complex terrain [3]. Other uses of squint can be envisioned, such as filling in shadow areas, or illuminating adjacent range subswaths at different times as the platform passes the desired area: in general, increasing the flexibility with which a particular area on the surface can be imaged within a single pass of the platform. Also, the application of squint is suited to a spaceborne platform, where the flight direction over the surface is determined by the orbit and cannot be changed significantly.

Section II describes the general SAR geometry, the notation, and the first part of equations describing signal properties at high squint. Section III completes the properties by describing squint and squinted beamwidth in terms of yaw, pitch, and elevation angle. Also, the yaw and pitch required to minimize Doppler centroid variation and maintain a range-independent azimuth bandwidth are derived, and residual Doppler centroid variation with antenna pointing errors are presented. Section IV discusses the SAR constraint of fitting the received echo between transmitted pulses in the context of high squint, and a fundamental limit on squint is shown. Section $\mathrm{V}$ discusses the 2-D spectrum of the SAR signal at high squint and considerations for processing. Finally, Section VI gives the conclusions.

\section{General Properties}

Fig. 1 illustrates the general spaceborne SAR geometry for a high squint angle. The platform position at azimuth time, $\eta$, is shown, as is the velocity vector $\mathbf{v}$. The velocity is assumed to be relative to the scatterer, so it includes the effect of earth rotation, and the plane perpendicular to $\mathbf{v}$ is the zero-Doppler plane. A scatterer is located by the azimuth time of closest approach, assumed $\eta=0$, and the closest approach range, $r_{0}$. The vector from the platform to the scatterer is $\mathbf{R}\left(\eta ; r_{0}\right)$, and 


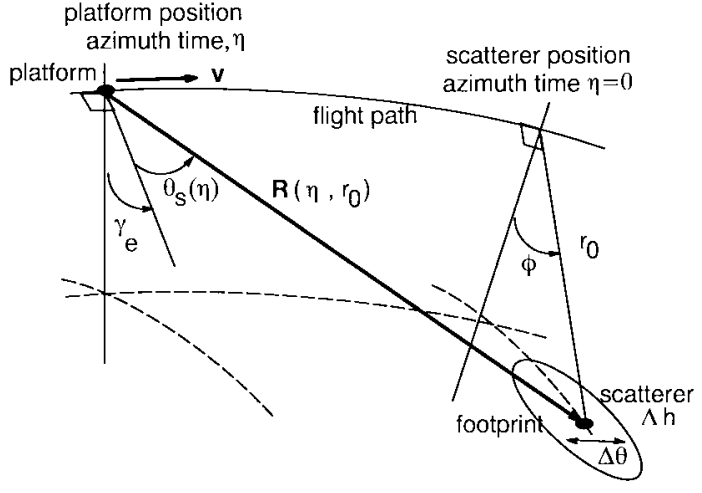

Fig. 1. Squint mode SAR imaging geometry.

together with $\mathbf{v}$ forms a slant range plane at the azimuth time, $\eta$. The magnitude of $\mathbf{R}\left(\eta ; r_{0}\right)$, which is the azimuth-varying distance from the platform to the scatterer, $R\left(\eta ; r_{0}\right)$, is known from the orbital data and earth model [4].

It is useful to define an instantaneous squint angle to the scatterer, $\theta_{s}(\eta)$, which is measured within the slant range plane, from the zero Doppler plane to $\mathbf{R}\left(\eta ; r_{0}\right)$. The instantaneous squint angle varies with azimuth, and is equal to the antenna boresight squint angle, $\theta$, when the scatterer is at the azimuth beam center. Also, the change in instantaneous squint angle to the scatterer, as the scatterer passes through the footprint, will be called the squinted beamwidth, $\Delta \theta$. The squint angle, $\theta$, and the squinted beamwidth are basic parameters that determine signal properties, and will be related to platform attitude and elevation angle in the next section.

In the SAR signal received from a point scatterer, the varying distance to a scatterer, with respect to the wavelength, causes an azimuth-varying phase in the signal. This results in an instantaneous azimuth (Doppler) frequency, $f_{\eta}$, which is related to the squint angle by [5]

$$
f_{\eta}=\frac{2 v \sin \left(\theta_{s}(\eta)\right)}{\lambda}
$$

where $v$ is the magnitude of the velocity vector, and $\lambda$ is the wavelength. The center of signal energy in the azimuth frequency domain is the Doppler centroid, which is the azimuth frequency when the scatterer is at the center of the beam

$$
f_{\eta c}=\frac{2 v \sin (\theta)}{\lambda} \text {. }
$$

Also, the azimuth bandwidth, $\Delta f_{\eta}$, is the change in azimuth frequency in the received signal as the scatterer passes through the beam, and so for narrow beamwidths can be expressed as a function of the squinted beamwidth as

$$
\Delta f_{\eta}=\frac{2 v \Delta \theta \cos (\theta)}{\lambda} .
$$

The squinted beamwidth determines the azimuth illumination time (synthetic aperture) of the scatterer, $\Delta \eta$. To find a relationship between squinted beamwidth and illumination time, we start with

$$
\sin \left(\theta_{s}(\eta)\right)=\frac{-1}{v} \frac{d R\left(\eta ; r_{0}\right)}{d \eta}
$$

which can be shown from the definition of the squint angle. Expanding both sides and rearranging gives

$$
\Delta \eta=\frac{v \cos (\theta) \Delta \theta}{\left|R^{\prime \prime}\left(\eta_{c} ; r_{0}\right)\right|}
$$

where $\eta_{c}$ is the azimuth time at which the scatterer is at beam center. The beam center time can be found from (4) with $\theta_{s}\left(\eta_{c}\right)=\theta$.

Finally, the elevation angle is measured in a plane perpendicular to the orbital plane. For the low squint case the usual elevation angle is measured at the scatterer's azimuth position, $\eta=0$, and is indicated by $\phi$ in Fig. 1. The elevation angle to the scatterer depends on the closest approach range, $r_{0}$, and the terrain height relative to an earth model, $\Delta h$. However, it will be convenient to define an elevation angle that is measured at the platform position, indicated by $\gamma_{e}$ in the figure. This is the angle between the orbital plane and the slant range plane. From Fig. 1, it can be shown that $\gamma_{e}$ is related to $\phi$ by

$$
\sin \left(\gamma_{e}\right)=\frac{r_{0}}{R\left(\eta ; r_{0}\right) \cos \left(\theta_{s}(\eta)\right)} \sin (\phi)
$$

so $\gamma_{e}$ is also related to the closest approach range and terrain height of the scatterer. As an example, for a circular orbit and spherical earth, the relationship between $\phi$, closest approach range, $r_{0}$, and terrain height, $\Delta h$, can be shown to be

$$
\sin (\phi)=\frac{\left(r_{e}+\Delta h\right) \sin \left(\alpha_{r}\left(r_{0}, \Delta h\right)\right)}{r_{0}}
$$

where

$$
\alpha_{r}\left(r_{0}, \Delta h\right)=\arccos \left[\frac{\left(r_{e}+\Delta h\right)^{2}+H^{2}-r_{0}^{2}}{2\left(r_{e}+\Delta h\right) H}\right]
$$

is the angle from the orbital plane to the scatterer, measured from earth center, $r_{e}$ is the nominal earth radius, and $H$ is the radius of the orbit.

\section{Platform Attitude}

\section{A. Squint and Squinted Beamwidth}

Signal properties depend on the squint and the squinted beamwidth as described above. In strip-map SAR, where the elevation beamwidth is typically much larger than the azimuth beamwidth, the orientation of the antenna footprint on the surface determines the variation of squint angle with elevation, and the squinted beamwidth. The footprint orientation in turn depends on the yaw and pitch rotations of the platform used to achieve squint.

To describe the squint angle, it is first necessary to define the coordinate system for describing the platform attitude, since the resulting equations depend on the coordinate system and on the order of the rotations. For the purpose of describing signal properties, the platform is assumed to be initially aligned to a zero-Doppler coordinate system, such as would be achieved in a yaw-steering mode like that of the ERS-1/2 platform [6]. This is shown in the first illustration in Fig. 2, where the $x$-axis is in the direction of the velocity vector of the platform relative to the earth, the $y$-axis is perpendicular to the orbital plane, and the $z$-axis completes the orthogonal 


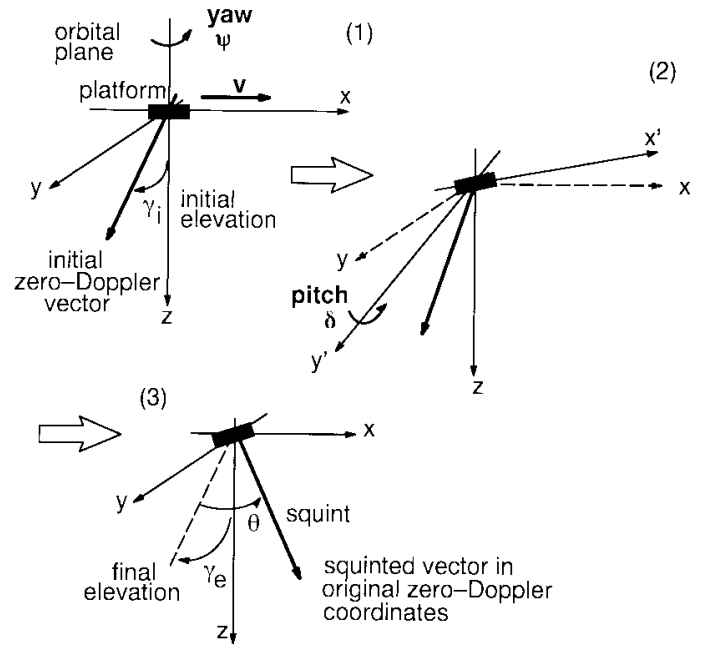

Fig. 2. Definition of yaw and pitch rotations from zero-Doppler coordinate system.

set. For purposes of describing the squint angle, only yaw and pitch rotations are described, since the results are to be described for any elevation angle. The first illustration shows an initial zero-Doppler vector at an initial elevation angle, $\gamma_{i}$. The yaw rotation, $\psi$, is assumed to be done first and is a rotation about the $z$-axis. Then, to correspond to the way attitude is controlled, the pitch rotation, $\delta$, is a rotation about the yaw-rotated $y^{\prime}$-axis of the platform, as shown in the second illustration in the figure. Both yaw and pitch are defined to be positive in the direction that creates a positive squint angle. The final rotated vector can be expressed in the original zeroDoppler coordinate system, as shown in the last illustration, having a squint angle, $\theta$, and a final elevation angle, $\gamma_{e}$. The squint and final elevation can be expressed as functions of $\gamma_{i}, \psi$, and $\delta$, as follows

$$
\begin{aligned}
\sin (\theta) & =\sin \left(\gamma_{i}\right) \sin (\psi)+\cos \left(\gamma_{i}\right) \sin (\delta) \cos (\psi) \\
\tan \left(\gamma_{e}\right) & =\frac{\tan \left(\gamma_{i}\right) \cos (\psi)-\sin (\delta) \sin (\psi)}{\cos (\delta)} .
\end{aligned}
$$

Also, for a given yaw and pitch there is a relationship between the initial and final elevation angles, so the initial elevation can be expressed as

$$
\tan \left(\gamma_{i}\right)=\frac{\tan \left(\gamma_{e}\right) \cos (\delta)+\sin (\delta) \sin (\psi)}{\cos (\psi)} .
$$

Finally, from the definitions of squint, elevation, yaw and pitch angles, these angles can be assumed to satisfy the following conditions

$$
\begin{array}{ll}
\text { squint } & -\pi / 2<\theta<\pi / 2 \\
\text { elevation } & 0<\gamma_{i}, \gamma_{e}<\pi / 2 \\
\text { yaw } & -\pi / 2<\psi<\pi / 2 \\
\text { pitch } & -\pi / 2<\delta<\pi / 2 .
\end{array}
$$

The squinted beamwidth is the difference in instantaneous squint angle, seen by a scatterer at the leading and trailing azimuth edges of the beam, $\theta_{+}$and $\theta_{-}$, respectively. Thus, $\Delta \theta=\left(\theta_{+}-\theta_{-}\right)$, and for narrow beamwidths this is

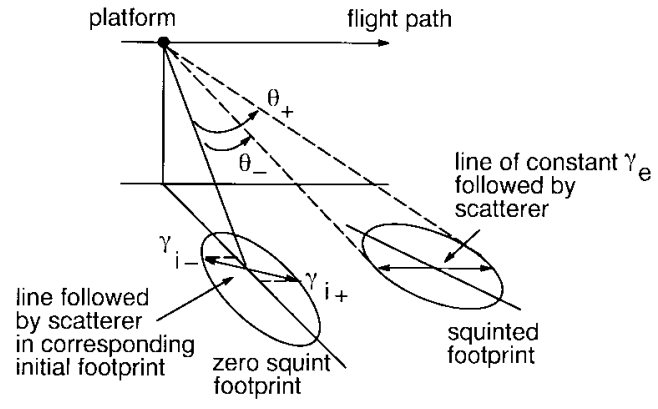

Fig. 3. Squinted beamwidth. Illustration of elevation angles at beam edges before and after squinting.

approximately

$$
\Delta \theta=\frac{\sin \left(\theta_{+}\right)-\sin \left(\theta_{-}\right)}{\cos (\theta)} .
$$

To find an expression for squinted beamwidth, start with initial vectors that point to the leading and trailing azimuth edges of the zero squint footprint and apply the yaw and pitch rotations to get the squinted vectors at the leading and trailing edges of the squinted footprint. The instantaneous squint angles of the initial vectors, before rotation, are approximately $\pm \lambda /(2 L)$, where $L$ is the azimuth antenna length. After rotation, the vectors should be aligned in such a way that a scatterer passes through the squinted footprint at a constant elevation angle, $\phi$. At the leading and trailing edges of the beam this corresponds to final elevation angles $\gamma_{e+}$ and $\gamma_{e-}$, from (10). However, calculations with typical SAR parameters showed that the change in $\gamma_{e}$ across the beam was so small that it, too, can be considered constant. Thus, for the initial vectors to get rotated to the line of constant $\gamma_{e}$ in the squinted footprint, the initial elevation angles are $\gamma_{i+}$ and $\gamma_{i-}$, as shown in Fig. 3. Applying the yaw and pitch rotation to these initial vectors, and using the relationship between initial and final elevation angles, gives

$$
\begin{aligned}
& \tan \left(\gamma_{i \pm}\right) \\
& \approx \frac{\tan \left(\gamma_{e}\right) \cos \left(\delta \pm \frac{\lambda}{2 L \cos \left(\gamma_{i}\right)}\right)+\sin (\psi) \sin \left(\delta \pm \frac{\lambda}{2 L \cos \left(\gamma_{i}\right)}\right)}{\cos (\psi)}
\end{aligned}
$$

where a narrow beamwidth has assumed, and the leading ("+") and trailing ("-") vectors are described using the \pm notation.

Also obtained from the rotations of these initial vectors are expressions for $\sin \left(\theta_{+}\right)$and $\sin \left(\theta_{-}\right)$. Substituting into (13) and rearranging gives the expression for squinted beamwidth

$$
\Delta \theta \approx \frac{\partial \theta}{\partial \gamma_{i}}\left[\gamma_{i+}-\gamma_{i-}\right]+\frac{\lambda \cos (\psi) \cos (\delta)}{L \cos (\theta)} .
$$

Equations (9) and (15) give the squint and squinted beamwidth, and hence the Doppler centroid, azimuth bandwidth, and exposure time, for a scatterer at the given elevation. In particular, the general expression for squinted beamwidth is seen to depend on squint and elevation angle, which is not normally assumed in SAR, and the effect on azimuth bandwidth will be seen in the next subsection. 


\section{B. Attitude Control}

The dependence of squint on elevation means that the squint angle, and hence Doppler centroid, will vary across the elevation beamwidth, resulting in the variation of Doppler centroid with range and terrain height. It is known that correct choice of yaw and pitch can minimize the variation of Doppler centroid with range, by aligning the footprint with an isoDoppler line [7]. Here, we present the solution for yaw and pitch to minimize the variation of squint with elevation, without small angle approximations, thus minimizing Doppler centroid variation with range and terrain height for any earth geometry. The solution for the yaw and pitch is evaluated at a nominal elevation angle corresponding to midswath and an average terrain height. The residual Doppler centroid variation, including the effect of errors in yaw and pitch, is then presented.

The rate of change of squint with elevation is minimized by setting

$$
\frac{\partial \theta}{\partial \gamma_{e}}=0
$$

By making use of the conditions in (12), noting that $\partial \gamma_{i} / \partial \gamma_{e}$ is nonzero and finite, it can be shown that to minimize squint variation with elevation it is sufficient to satisfy

$$
\frac{\partial \sin (\theta)}{\partial \gamma_{i}}=0
$$

Evaluating this equation using (9) gives

$$
\tan (\psi)=\tan \left(\gamma_{i}\right) \sin (\delta) .
$$

Thus, given a desired squint angle, $\theta_{d}$, and a nominal final elevation angle, $\gamma_{e d},(9),(10)$, and (11) can be written in terms of the three unknowns: yaw, pitch, and a nominal initial elevation angle. These equations can be solved for the required $\psi, \delta$, and $\gamma_{i}$ to give

$$
\begin{aligned}
\tan (\psi) & =\sin \left(\gamma_{e d}\right) \tan \left(\theta_{d}\right) \\
\sin (\delta) & =\cos \left(\gamma_{e d}\right) \sin \left(\theta_{d}\right) \\
\tan \left(\gamma_{i}\right) & =\tan \left(\gamma_{e d}\right) / \cos \left(\theta_{d}\right) .
\end{aligned}
$$

Once $\psi, \delta$, and $\gamma_{i}$ are found, the procedure for achieving the desired squint and elevation is as follows: Start with the platform aligned to the zero-Doppler coordinate system, with the antenna pointed electronically or mechanically in elevation to the required $\gamma_{i}$ at midswath, then apply the yaw and then the pitch rotations given by the solutions for $\psi$ and $\delta$.

Assuming the yaw and pitch angles are chosen to minimize squint variation with elevation, an expression for the squint angle which is independent of elevation angle can be found. Substituting for $\gamma_{i}$ from (18) into the expression for squint in (9), and rearranging, gives

$$
\cos (\theta)=\cos (\psi) \cos (\delta) .
$$

To find the residual Doppler centroid variation for various squint angles, the optimum yaw and pitch angles were calculated for an elevation angle at midswath and nominal terrain height, and antenna pointing errors were added. Then, the elevation angles were calculated at the edges of the swath,
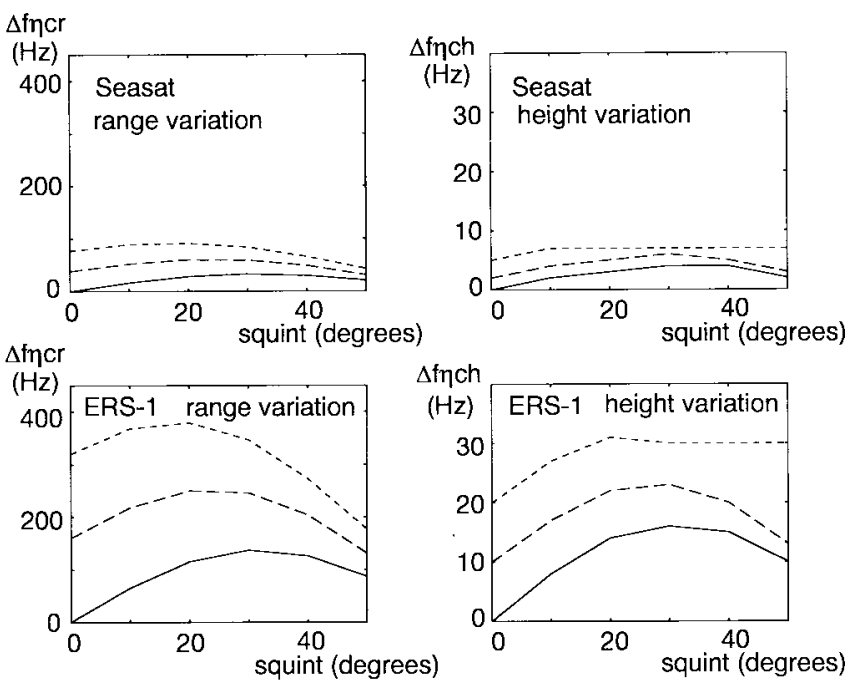

Fig. 4. Doppler centroid error versus squint, due to range variation and 1000 $\mathrm{m}$ height variation, for Seasat (L-band) and ERS-1 (C-band) parameters. Solid curve indicates $0^{\circ}$ antenna pointing error, dashed curve indicates $\pm 0.5^{\circ}$ error, and dotted curve indicates $\pm 1^{\circ}$ error.

and with a terrain height change of $\Delta h=1000 \mathrm{~m}$. This was used to find the difference in squint angle from the desired squint angle, which in turn was used to find the residual Doppler centroid error. The parameters of the calculations were chosen to be representative of the platforms, Seasat, ERS-1, and X-SAR, with wavelengths at L-band, C-band, and $\mathrm{X}$-band, respectively. For the satellite platforms, Seasat and ERS-1, the altitude is $800 \mathrm{~km}$ and the ground swath width is $100 \mathrm{~km}$, and the nominal elevation angle at midswath is $21^{\circ}$. For the shuttle platform, X-SAR, the altitude is 225 $\mathrm{km}$ and the ground swath width is $40 \mathrm{~km}$. Also, for X-SAR, cases of near and far incidence are considered, corresponding to the nominal elevation angles of 21 and $45^{\circ}$, respectively. Figs. 4 and 5 show the residual Doppler centroid error, in Hertz, versus squint angle in degrees for the different cases. Results are given for different values of maximum antenna pointing error: $0^{\circ}$ error, $\pm 0.5^{\circ}$ error, and $\pm 1^{\circ}$ error. For a given maximum antenna pointing error, the Doppler centroid error corresponding to the worst case combination of yaw and pitch is presented.

Variations in Doppler centroid should be less than (PRF PBW), where PBW is the processed azimuth bandwidth, so that the Doppler centroid variation should typically be less than about 200 to $300 \mathrm{~Hz}$ for spaceborne platforms. It is possible to accommodate some range variation of Doppler centroid during processing in the two-dimensional frequency domain, but a significant variation of Doppler centroid with terrain height will lead to azimuth ambiguities that cannot be easily accommodated in processing.

As can be seen in Figs. 4 and 5, the proper choice of yaw and pitch angles allows the collection of SAR data with acceptable Doppler centroid variation in most cases. Antenna pointing errors increase the residual Doppler centroid variation, although the variation is almost as large at nominally zero squint as it is at higher squint angles. The residual Doppler centroid variation actually decreases with squint angles past 

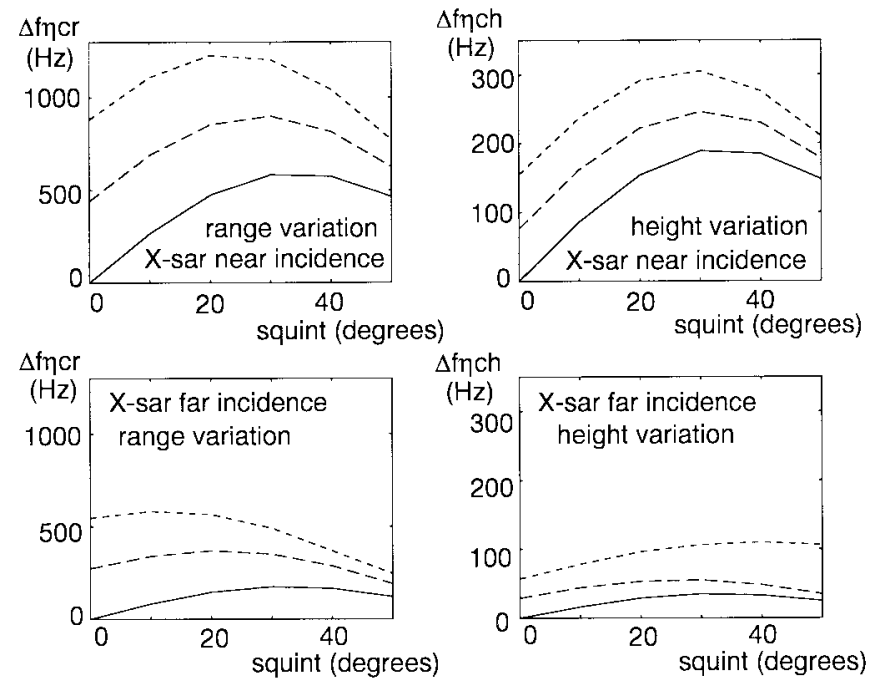

Fig. 5. Doppler centroid error versus squint, due to range variation and 1000 $\mathrm{m}$ height variation, for X-SAR (X-band) parameters at near incidence $\left(21^{\circ}\right)$ and far incidence $\left(45^{\circ}\right)$. Solid curve indicates $0^{\circ}$ antenna pointing error, dashed curve indicates $\pm 0.5^{\circ}$ error, and dotted curve indicates $\pm 1^{\circ}$ error.

about $30^{\circ}$, because Doppler centroid is proportional to $\sin (\theta)$. The Doppler centroid variation with range and terrain height at L-band (Seasat) is negligible even with a $1^{\circ}$ antenna pointing error. At C-band (ERS-1) the range variation is barely acceptable with a $0.5^{\circ}$ pointing error, and would require some accommodation in processing in the case of a $1^{\circ}$ pointing error. The terrain height variation of Doppler centroid with ERS-1 parameters is acceptable. With the small X-band wavelength, Doppler centroid variation is quite large, especially for the near incidence case. Also, the terrain height variation of Doppler centroid is larger for X-SAR because of the lower altitude of the space shuttle. The X-SAR, near incidence case represents the worst case considered, and the terrain height variation is possibly unacceptable in mountainous terrain. At far incidence, the Doppler centroid variation is smaller, so that the terrain height variation becomes acceptable, although the range variation would require some accommodation at a $1^{\circ}$ pointing error.

Next, the squinted beamwidth can be found in the case that attitude control is used to minimize Doppler centroid variation. In this case, the first term in (15) is negligible, and from the relationship in (22) the expression for squinted beamwidth reduces to

$$
\Delta \theta=\frac{\lambda}{L}
$$

independent of squint and elevation angle. By maintaining a constant squinted beamwidth of $\lambda / L$, the bandwidth becomes

$$
\Delta f_{\eta}=\frac{2 v \cos (\theta)}{L}
$$

which is independent of range and decreases as the cosine of the squint angle. Thus, proper selection of yaw and pitch angles not only minimizes Doppler centroid variation, but also preserves the property of SAR imaging that the azimuth bandwidth is independent of range. Also, the dependence of
TABLE I

Azimuth Bandwidth Versus Squint for X-SAR Parameters: YaW Rotation ONLY; AND With Optimal YaW and Pitch

\begin{tabular}{|c|c|c|c|}
\hline & \multicolumn{3}{|c|}{ azimuth bandwidth $(\mathrm{Hz})$} \\
\hline squint & \multicolumn{2}{|c|}{ yaw only } & yaw and pitch \\
\hline (degrees) & near incidence & far incidence & \\
\hline 0 & 1300 & 1300 & 1300 \\
10 & 1404 & 1298 & 1280 \\
20 & 1629 & 1285 & 1222 \\
30 & 1827 & 1245 & 1126 \\
40 & 1893 & 1157 & 996 \\
50 & 1766 & 995 & 836 \\
\hline
\end{tabular}

azimuth bandwidth on $\cos (\theta)$ will prove useful with respect to SAR imaging constraints in the next section.

To illustrate the effect of the use of optimal yaw and pitch angles on the azimuth bandwidth, Table I shows the azimuth bandwidth as a function of squint angle for various cases. First, the bandwidth was calculated by assuming that only a yaw rotation was assumed to achieve the squint. Results are given for the near and far incidence cases of the X-SAR platform, corresponding to different ranges for the same platform altitude. For the same squint angle, the difference in bandwidth between near and far incidence cases is significant. Also, at near incidence the azimuth bandwidth can be significantly larger, requiring a large PRF that would reduce the available swath width for the given SAR parameters. In contrast, the table also shows the bandwidth when yaw and pitch angles are optimized to minimize the variation of squint with elevation. In this case, the results for the near and far incidence cases were the same, and agreed with the expression for bandwidth in (24).

\section{SAR CONSTRAINT}

A fundamental constraint in SAR imaging is that a received echo must fit between consecutive transmitted pulses. The echo length depends on the swath width and squint angle, and the PRF is the azimuth sampling rate which must be greater than the azimuth bandwidth. It is known that this constraint requires a trade-off between swath width and azimuth resolution and sets a minimum area for the antenna [1]. In this section, the effect of a high squint angle is investigated.

The range swath width of the scene, $\Delta r$, is the difference in closest approach range to scatterers at each end of the image swath. The duration of a received echo is the time delay difference between the nearest and farthest scatterers seen by the radar, $2 \Delta R / c$ where $c$ is the speed of light, plus the transmitted pulse length, $T$, since the scatterer distribution is convolved with the pulse. Without loss of generality, assume a forward squint angle, so the largest delay corresponds to a scatterer at far range and at the leading edge of the beam, while the smallest delay corresponds to a scatterer at near range at the trailing edge of the beam. The time between pulses must be long enough to contain the transmitted pulse, the received 


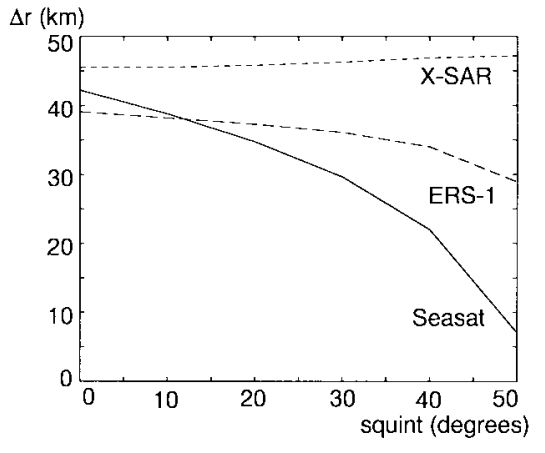

Fig. 6. Closest approach range swath versus squint for Seasat (L-band), ERS-1 (C-band), and X-SAR (X-band).

echo, and a guard space, $T_{\mathrm{sp}}$, that allows for variations in the range delay of the signal and to allow some flexibility in choosing the PRF. This constraint can be expressed as

$$
\frac{2 \Delta R}{c}+2 T+T_{\mathrm{sp}}<\frac{1}{\mathrm{PRF}} .
$$

For a given closest approach range swath, $\Delta r$, the length of the received echo increases with squint, as $1 / \cos (\theta)$, due to the fact that with squint, the distance along the look direction between the near and far ends of the swath increases. If the yaw and pitch angles are chosen such that the azimuth bandwidth is given by (24), then the PRF can be allowed to decrease with squint as $\cos (\theta)$, allowing a greater time between pulses. In addition, however, the received echo length is further increased with squint because of increased range migration, or change in delay to the scatterer over the exposure time.

To describe the effect of squint, consider the approximate expression for $\Delta R$

$$
\Delta R \approx \frac{\Delta r}{\cos (\theta)}+\frac{\lambda r_{\text {ref }} \tan (\theta)}{L \cos (\theta)}
$$

where the first term is due to the viewing angle and the second term is due to range migration. To accommodate the decrease of azimuth bandwidth with squint angle, let PRF $=\mathrm{PRF}_{0} \cos (\theta)$, where $\mathrm{PRF}_{0}$ is the the value at zero squint. Also, let the guard space be proportional to the time between pulses, so $T_{\mathrm{sp}}=T_{\mathrm{sp} 0} / \cos (\theta)$, where $T_{\mathrm{sp} 0}$ is the value at zero squint. By substituting these expressions into (25), the maximum closest approach range swath that can be imaged for a given squint angle can be shown to be

$$
\begin{aligned}
\Delta r< & \frac{c}{2}\left[\frac{1}{\mathrm{PRF}_{0}}-\left(2 T+T_{\mathrm{sp} 0}\right)\right] \\
& +\left[c T(1-\cos (\theta))-\frac{\lambda r_{\mathrm{ref}}}{L} \tan (\theta)\right]
\end{aligned}
$$

where the first term is the swath width at zero squint. As squint increases, the allowable swath width depends on the opposing effects represented by the last two terms in this equation. Without range migration, as the PRF decreases with increasing squint there is proportionally more room for the received echo since the transmitted pulse length stays the same. However, the increase in range migration with squint, increases the echo length and reduces the allowable swath width.

This was investigated by calculating the maximum available swath width as a function of squint angle. $\Delta R$ was calculated by finding $R\left(\eta ; r_{0}\right)$ at the closest and farthest points in the

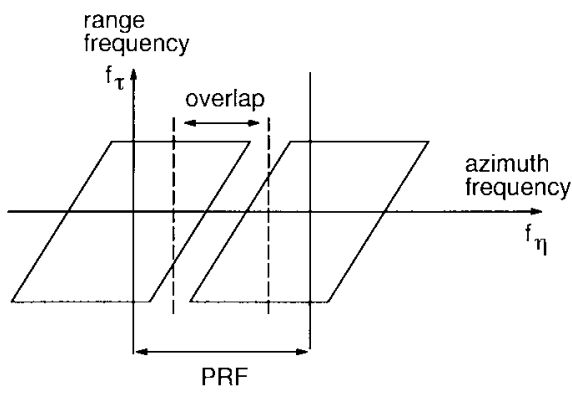

Fig. 7. Skewed region of support of data spectrum.

swath, for spaceborne SAR geometry using parameters for Seasat, ERS-1, and X-SAR (near incidence). The maximum value of closest approach range swath, $\Delta r$, for which the condition in (25) is satisfied was found. The PRF was calculated for a given squint angle, using the azimuth bandwidth in (24) and an oversampling rate of 1.2. The guard space was set be $T_{\mathrm{sp}}=0.4 / \mathrm{PRF}$, which is relatively large, but gives range swath limits at zero squint that are equal to the typical range swaths of about $40 \mathrm{~km}$.

The results are shown in Fig. 6. For Seasat, the allowable range swath width decreases significantly with squint, and becomes too small to be practical for squint angles above about $40^{\circ}$. For the smaller wavelength of ERS-1, the allowable swath width is larger, but still decreases noticeably with squint. For these cases it is seen how, depending on wavelength, the increased echo length due to range migration gives a fundamental limitation on squint angle. The X-SAR platform has a smaller wavelength and lower altitude, resulting in a small amount of range migration so that the allowable swath width increases slightly with squint.

\section{DATA SPECTRUM}

Assuming precision processing in the 2-D frequency domain, at high squint the region of support of the data spectrum poses an interesting problem, which arises from the dependence of the Doppler centroid on range frequency, $f_{\tau}$, in the raw data. To see this, note that the relationship between Doppler centroid and squint angle in (2) was defined at the carrier frequency, $f_{0}=c / \lambda$. In the 2-D frequency domain, however, the Doppler centroid can be determined for each range-frequency component as [8]

$$
f_{\eta c}=\frac{2 v\left(f_{0}+f_{\tau}\right) \sin (\theta)}{c}
$$

This results in a skewed region of support of the data spectrum, as shown in Fig. 7. Also, because of the sampling of the azimuth signal, this skew can cause parts of the spectrum to cross into the adjacent PRF band. Note that since all the repeated spectra are skewed the same way, there is no actual aliasing of signal energy at this point. However, after range cell migration correction and inverse transformation to the range-Doppler domain, the signal energy from the repeated spectra are all aligned in the same range bin, and the corrected trajectories interfere with each other. This results in aliasing in azimuth-frequency, and prevents the application of the azimuth compression filter over all the azimuth frequencies in the signal. Finally, the PRF cannot generally be increased 


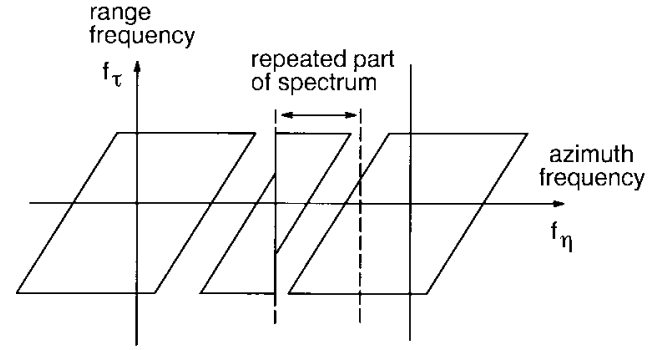

Fig. 8. Replication of parts of spectrum to expand azimuth bandwidth.

enough to alleviate this problem, because of the SAR imaging constraints discussed earlier. Thus, while the SAR data can be collected with an azimuth sampling rate that decreases with squint angle, the processing of the data into an image requires extra azimuth bandwidth to avoid aliasing. This problem occurs for any SAR processing algorithm, since the potential for aliasing is inherent in the signal.

This problem can be accommodated during processing by replicating the parts of the spectrum that overlap, as illustrated in Fig. 8. This produces a 2-D spectrum without overlap, removing the potential for aliasing during processing. Essentially, the azimuth bandwidth is expanded to accommodate the nature of the squinted SAR signal. Also, this replication of range lines can be done in the range-Doppler domain, before the range Fourier transform. This allows all processing steps that depend on azimuth-frequency, such as range-migration correction, to be performed correctly for all azimuth-frequencies in the signal. Then, the unwanted pieces of duplicated spectrum, shown in the figure, can be removed in the 2-D frequency domain by applying a window.

\section{CONCLUSION}

General properties of spaceborne, strip-map SAR which are valid for very large squint angles have been presented in terms of the squint and the squinted beamwidth, which are in turn expressed in terms of the platform attitude and elevation angle. The yaw and pitch angles which minimize the variation of Doppler centroid with range and terrain height have been derived, and calculation of the residual Doppler centroid variation in the presence of antenna pointing errors for Seasat, ERS-1, and X-SAR parameters have been shown to give acceptable results, except for the case of X-SAR at near incidence. It was shown that the correct yaw and pitch are also necessary to maintain the SAR property of an azimuth bandwidth that is independent of range. In this case, the azimuth bandwidth of the SAR data decreases as the cosine of the squint angle. The smaller PRF obtained with proper steering helps to satisfy the SAR constraint of fitting a received echo between transmitted pulses. However, because of the increased range migration at higher squint, this SAR constraint places a fundamental limit on the squint angle for imaging a given swath width. Finally, the variation of the Doppler centroid with range frequency results in a skewed region of support of the 2-D Fourier transform of the raw data, such that parts of the repeated spectra of the sampled signal can overlap and lead to aliasing during processing. Thus, it may be necessary to replicate data at certain azimuth frequencies to avoid overlap.
Although squint mode has not been previously used in spaceborne, strip-map SAR, it has been shown that with proper attitude control, such data can be collected accurately, such that typical SAR properties are preserved, and in way that allows efficient processing in the 2-D frequency domain.

\section{REFERENCES}

[1] R. O. Harger, Synthetic Aperture Radar Systems: Theory and Design New York: Academic, 1970

[2] I. G. Cumming, F. H. Wong, and R. K. Raney, "A SAR processing algorithm with no interpolation," in IGARSS'92 Proc., 1992, pp. 376-379.

[3] J. Way and E. A. Smith, "The evolution of synthetic aperture radar systems and their progression to the EOS SAR," IEEE Trans. Geosci. Remote Sensing, vol. 29, pp. 962-985, Nov. 1991.

[4] B. C. Barber, "Theory of digital imaging from orbital synthetic-aperture radar," Int. J. Remote Sensing, vol. 6, no. 7, pp. 1009-1057, 1985.

[5] R. K. Raney, "Doppler properties of radars in circular orbits," Int. J. Remote Sensing, vol. 7, no. 9, pp. 1153-1162, 1986.

[6] H. Runge, "Benefits of antenna yaw steering for SAR," in IGARSS'91 Proc., 1991, pp. 257-261.

[7] C. Y. Chang, M. Jin, and J. C. Curlander, "Squint mode SAR processing algorithms," in Proc. IGARSS'89, 1989, pp. 1702-1706.

[8] R. Bamler and H. Runge, "PRF-ambiguity resolving by wavelength diversity," IEEE Trans. Geosci. Remote Sensing, vol. 29, pp. 997-1003, Nov. 1991.

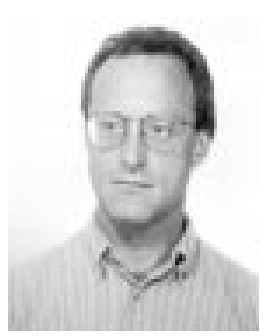

Gordon W. Davidson (S'83-M'86) received the B.Sc. in electrical engineering from the University of Calgary, Calgary, Alta., Canada, in 1984. He received the M.Eng. degree in systems and computer engineering from Carleton University, Ottawa, Ont. Canada, in 1986, with his thesis investigating adaptive equalization techniques on a fading channel. In 1994, he received the Ph.D. degree in electrical engineering from the University of British Columbia (UBC), Vancouver, B.C., Canada, for which he investigated squint mode SAR signal properties and developed high squint SAR processing based on the chirp scaling algorithm.

From 1986 to 1988, he was with Bell Northern Research, Ottawa, in software development, and in 1989 he was a research assistant in adaptive signal processing for communications at Carleton University. During his Ph.D. study he consulted for MacDonald Dettwiler in the area of SAR processing. In 1994 and 1995, he was a lecturer at UBC in digital signal processing. Then from 1995 to 1996, he was a guest scientist at the German Aerospace Research Establishment (DLR), Oberpfaffenhofen, Germany, working in ScanSAR and SAR interferometry. Since 1997, he has been in the Advanced Development Group at Glenayre, Vancouver, working in wireless communications.

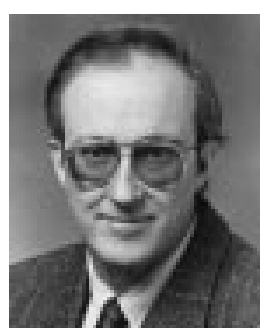

Ian Cumming (S'63-M'66) received the B.Sc. degree in engineering physics from the University of Toronto, Toronto, Ont., Canada, in 1961, and the $\mathrm{Ph} . \mathrm{D}$. degree in computing and automation from Imperial College, University of London, London, U.K., in 1968 .

Following work in steel mill automation and sonar signal processing, he joined MacDonald Dettwiler and Associates in 1977. Since then, he has developed synthetic aperture radar signal processing algorithms, and has worked on systems for processing polarimetric and interferometric radar data, and for the compression of radar data. In 1993, he joined the Department of Electrical Engineering, University of British Columbia, Vancouver, B.C., Canada, where he holds the MacDonald Dettwiler/NSERC Industrial Research Chair in radar Remote Sensing. The associated laboratory supports a research staff of ten engineers and students working in the fields of SAR processing, SAR data encoding, satellite SAR, two-pass interferometry, airborne, along-track interferometry, airborne polarimetric radar classification, and SAR Doppler estimation. 